\title{
Modeling and Scaling of the Viscosity of Suspensions of Asphaltene Nanoaggregates
}

\section{Rajinder Pal}

Department of Chemical Engineering, University of Waterloo, Waterloo, ON N2L 3G1, Canada; rpal@uwaterloo.ca; Tel.: +1-519-888-4567 (ext. 32985)

Academic Editor: Alireza Bahadori

Received: 27 February 2017; Accepted: 25 May 2017; Published: 1 June 2017

\begin{abstract}
The scaling and modeling of the viscosity of suspensions of asphaltene nanoaggregates is carried out successfully taking into consideration the solvation and clustering of nanoaggragates, and the jamming of the suspension at the glass transition volume fraction of asphaltene nanoaggregates. The nanoaggregates of asphaltenes are modeled as solvated disk-shaped "core-shell" particles taking into account the most recent small-angle neutron scattering (SANS), small-angle X-ray scattering (SAXS), and solid-state ${ }^{1} \mathrm{H}$ NMR studies available on the size and structure of asphaltene nanoaggregates. This work is an extension of our earlier studies on modeling of asphaltene suspensions where solvation of asphaltene nanoaggregates was not considered. A new mathematical model is developed for estimating the aspect ratio (ratio of thickness to diameter of particle) and the corresponding intrinsic viscosity of suspension of solvated disk-shaped asphaltene nanoaggregates using the experimental relative viscosity data of suspensions at low asphaltene concentrations. The solvation of asphaltene nanoaggregates is found to be significant. The intrinsic viscosity increases with the increase in the degree of solvation of nanoaggregates. At high concentrations of asphaltenes, clustering of solvated nanoaggregates dominates resulting in large viscosities. A new scaling law is discovered to scale the viscosity data of different asphaltene suspensions. According to the new scaling law, a unique correlation is obtained, independent of the type of asphaltene system, when the data are plotted as $\left(\eta_{r}-1\right) /[\eta]_{S}$ versus $\phi_{S}$ where $\eta_{r}$ is the relative viscosity of suspension, $[\eta]_{S}$ is the intrinsic viscosity of suspension of solvated nanoaggregates, and $\phi_{S}$ is the volume fraction of solvated nanoaggregates. Twenty sets of experimental viscosity data on asphaltene suspensions gathered from different sources are used to verify and confirm the scaling law and the viscosity model proposed in this work. Based on the experimental data, the glass transition volume fraction of solvated asphaltene nanoaggregates where jamming of suspension, and hence divergence of viscosity, takes place is found to be approximately 0.4 . The viscosity model proposed in this work can be used to predict the viscosity of a new asphaltene system over a broad range of asphaltene concentrations provided that the intrinsic viscosity of the suspension is obtained from viscosity measurements at very low asphaltene concentrations.
\end{abstract}

Keywords: asphaltenes; asphaltene nanoaggregates; viscosity; rheology; heavy oil; crude oil; hydrocarbon

\section{Introduction}

Asphaltenes are complex hydrocarbon molecules found in crude oils. An asphaltene molecule consists of a large polyaromatic hydrocarbon (PAH) ring system (5-10 fused rings) with peripheral alkanes [1,2]. As shown schematically in Figure 1, the asphaltene molecule has a flat island-type architecture dominated by aromatic ring system at the core with peripheral alkanes. The molecular weight of asphaltene is approximately $750 \mathrm{~g} / \mathrm{mol}[2]$. 


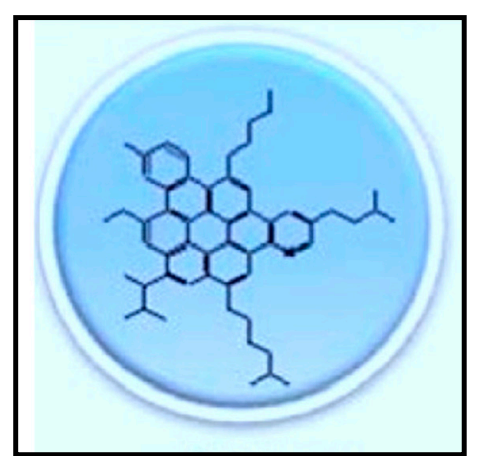

Figure 1. The asphaltene molecule consisting of PAH ring system at the core and alkanes at the periphery [2].

Asphaltene molecules have a strong tendency to aggregate even in good solvents. Precise SANS (small angle neutron scattering) and SAXS (small angle X-ray scattering) studies carried out by several different research groups [1-5] have proven that asphaltene molecules self-associate into nanoaggregates that have disk-shaped "core-shell" morphology consisting of a densely packed aromatic structure at the core with alkyl side chains as a shell. According to Eyssautier et al. [3], the self-association of molecular aromatic sheets into nanoaggregates begin at a very low asphaltene concentration, referred to as NAC (nanoaggregate concentration), usually in the range of 50 to $200 \mathrm{mg} / \mathrm{L}$. In a recent publication by Majumdar et al. [1] entitled "Insights into asphaltene aggregate structure using ultrafast MAS solid-state ${ }^{1} \mathrm{H}$ NMR spectroscopy", the authors highlight that "Nanoaggregation occurs through $\pi-\pi$ interactions, and clusters consist of a rigid central core and a mobile periphery".

In the oil field, asphaltenes are generally unwanted as they cause many problems in the production, transportation, and refining of crude oils. The high viscosity of heavy crude oils and bitumen is largely due to the presence of asphaltenes. They also tend to precipitate and adhere to surfaces causing blockage of pipelines (see Figure 2). Thus asphaltenes are oil pipeline equivalent of "bad cholesterol" that causes build-up of plaque in human arteries and blockage of arteries. Asphaltene precipitation is also known to alter the wetting characteristics of mineral surfaces within the reservoir resulting in low oil recovery efficiency. They also cause emulsion problems, as they tend to adsorb on to the oil/water interface and provide steric-stabilization of emulsion droplets. Emulsions with asphaltene nanoaggregates present at the oil/water interface are generally very stable and difficult to resolve.

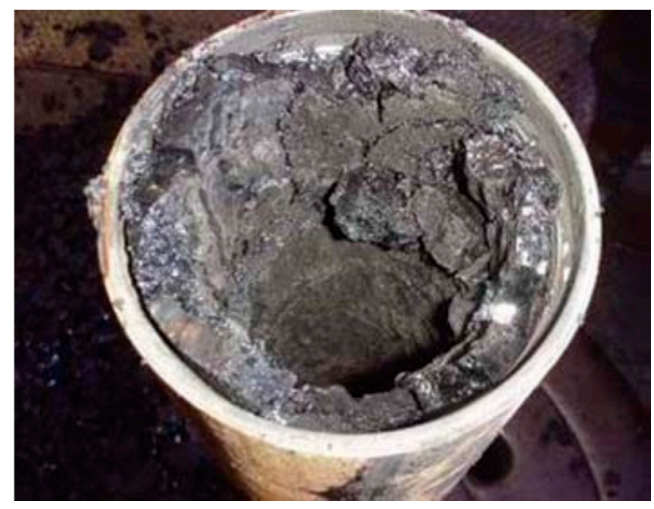

Figure 2. Clogging of a pipe by asphaltenes [6].

The modeling of the viscosity of asphaltene suspensions is a longstanding unresolved problem. The viscosity models originally developed for suspensions of spherical particles are often applied to suspensions of asphaltene nanoaggregates [7]. Now that the structure of asphaltene nanoaggregates 
is demystified, it is time to model the viscosity of suspensions of asphaltene nanoaggregates more accurately. In the design and operation of production, transportation, and processing facilities for crude oils, it is important to be able to predict the viscosity of crude oil accurately as a function of its asphaltene content.

In this article, the modelling and scaling of the viscosity of suspensions of asphaltene nanoaggregates is discussed in detail taking into consideration the most recent information available on the morphology of asphaltene nanoaggregates. A large pool (20 sets) of available experimental data on the viscosity of asphaltene suspensions is considered to verify the scaling and modelling analysis presented in this work. The experimental data covers different sources of asphaltenes, different types of asphaltenes, different types of solvents, and broad ranges of temperatures and volume fraction of asphaltenes. This work is an extension of our earlier work $[7,8]$ where asphaltene nanoaggregates were modelled as rigid disk-shaped particles ignoring the solvation of nanoaggregates. In the present work, the nanoaggregates are modelled as disk-shaped "core-shell" particles with solvation occurring in the shell portion of the nanoaggregates. To our knowledge, this is the first study to incorporate the most recent information on the morphology of nanoaggregates in the modeling and scaling of viscosity of asphaltene suspensions including the solvation effects. The concepts of jamming and glass transition in asphaltene suspension with the increase in the asphaltene content are also incorporated in the analysis for the first time.

\section{Microstructure of Asphaltene Suspensions}

\subsection{Size and Structure of Nanoaggregates}

Figure 3 shows schematically the size and structure of asphaltene nanoaggregates. As already pointed out, nanoaggregates are disk-shaped "core-shell" particles consisting of a rigid central core (densely packed aromatic structure) and a mobile periphery (alkyl side chains). The core has a diameter of $3.64 \mathrm{~nm}$ and the shell thickness is $1.44 \mathrm{~nm}$. The thickness of the disk is $0.67 \mathrm{~nm}$. These dimensions are based on the precise SANS measurement of asphaltene nanostructure by Eyssautier et al. [3].

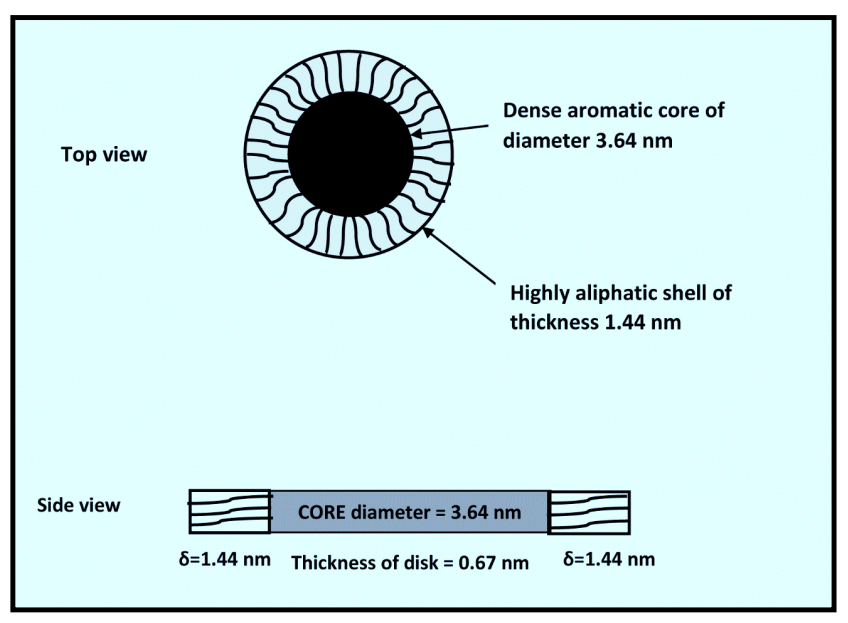

Figure 3. Size and structure of asphaltene nanoaggregate.

\subsection{Clustering of Nanoaggregates}

Clusters of asphaltene nanoaggregates are formed when the asphaltene concentration exceeds the critical cluster concentration (CCC) [2]. The CCC of asphaltenes is about 2 to $5 \mathrm{~g} / \mathrm{L}$. Figure 4 shows a schematic diagram of a cluster of asphaltene nanoaggregates. The clustering of nanoaggregates is driven by Brownian effect. Due to Brownian motion, the nanoaggregates come in contact with each other and form clusters. A significant amount of the continuous phase fluid is immobilized within the clusters leading to an increase in the effective volume fraction of the dispersed-phase. 


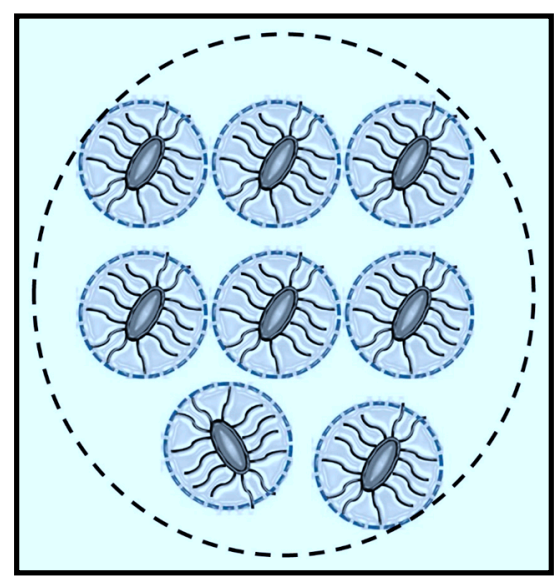

Figure 4. Cluster of asphaltene nanoaggregates.

\subsection{Phase Diagram of Asphaltene Suspensions}

A number of experimental and theoretical studies have been carried out on the equilibrium phase diagram of hard colloidal platelets [9-13]. Although these studies do not deal with suspensions of asphaltene nanoaggregates, they can be used to speculate the equilibrium phase behaviour of suspensions of disc-shaped asphaltene nanoaggregates. Based on these studies, Figure 5 exhibits the changes in the asphaltene suspension morphology expected upon progressively increasing the volume fraction of the disk-shaped asphaltene nanoaggregates under equilibrium conditions. For $\phi<\phi_{I N}$, the suspension is isotropic with random orientations and random positions of nanoaggregates where $\phi_{I N}$ is the volume fraction of particles where transition from isotropic phase to nematic phase takes place. For $\phi>\phi_{I N}$, the suspension becomes nematic liquid crystal with random positions and ordered orientations (note there may be a narrow transition region where isotropic and nematic phases co-exist). When $\phi$ exceeds $\phi_{N C}$, ordered crystals appear and we have a two-phase region where crystal + nematic phases co-exist. For $\phi>\phi_{C}$, the entire suspension becomes ordered columnar crystalline. According to Wensink and Lekkerkerker [9], the columnar order becomes stable only beyond a $\phi$ value of approximately 0.40. However, it should be emphasized that there is no experimental proof yet available in the literature to show that asphaltene suspensions follow the equilibrium phase behaviour as shown in Figure 5.

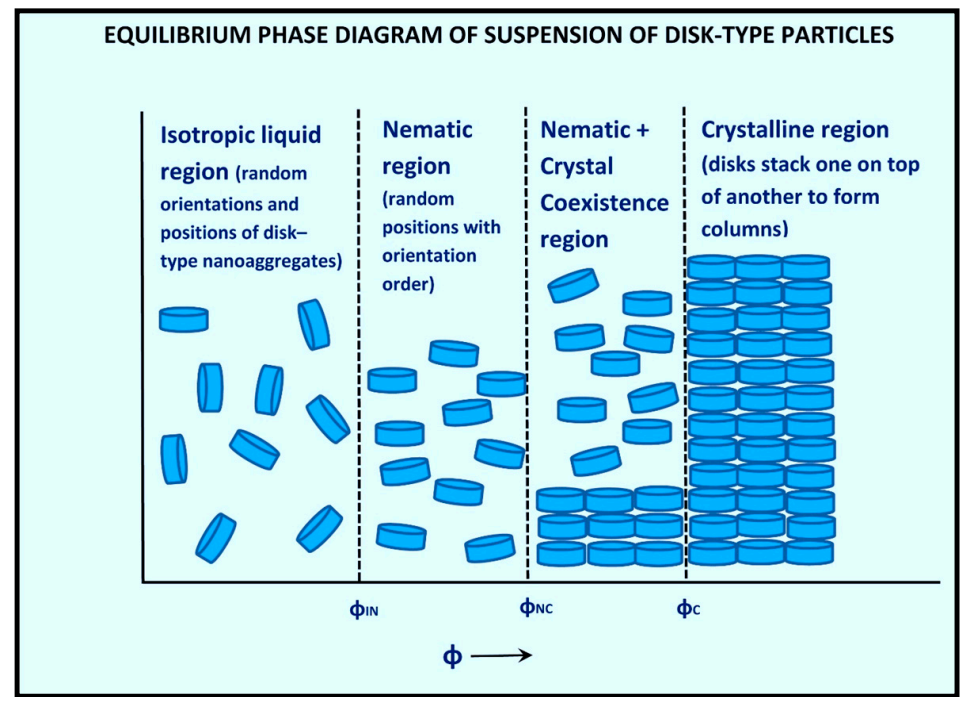

Figure 5. Changes in suspension morphology expected with increase in $\phi$ under equilibrium conditions. 
In order to observe the equilibrium phase behaviour depicted in Figure 5, it is essential that the suspension is concentrated (quenched) very slowly or quasi-statically such that the suspension is at equilibrium at all times. A different picture is expected if the suspension is concentrated rapidly, that is, under non-equilibrium conditions. Figure 6 shows the expected non-equilibrium phase diagram of asphaltene nanosuspensions. When the volume fraction of particles is increased, that is, $1 / \phi$ is decreased, the suspension is initially fluidic with well-dispersed particles. The particles have random orientations and random positions. As $\phi$ is increased further $(1 / \phi$ is decreased further) from dilute to nondilute condition, the rotation and translation of the particles becomes increasingly restricted as the availability of free space to the particles becomes less and less. The particles become crowded and are caged temporarily by their neighbours. There exists some orientation order in these temporary clusters or cages. The temporary clusters are continually formed and destroyed by thermal excitations. The size and number of clusters increase with the increase in $\phi$ although the suspension remains ergodic at moderate concentrations of particles. As the suspension is concentrated further $(1 / \phi$ is decreased further), the glass transition volume fraction $\phi_{g}$ is reached where each particle gets trapped in a permanent cage and the suspension as a whole is jammed into disordered glassy state. Some orientation order is expected in micro-domains of the glassy state. Due to jamming of particles, the glassy state is expected to exhibit a finite yield-stress. For a glassy suspension to flow, it is necessary to apply a shear stress exceeding the yield-stress of the suspension.

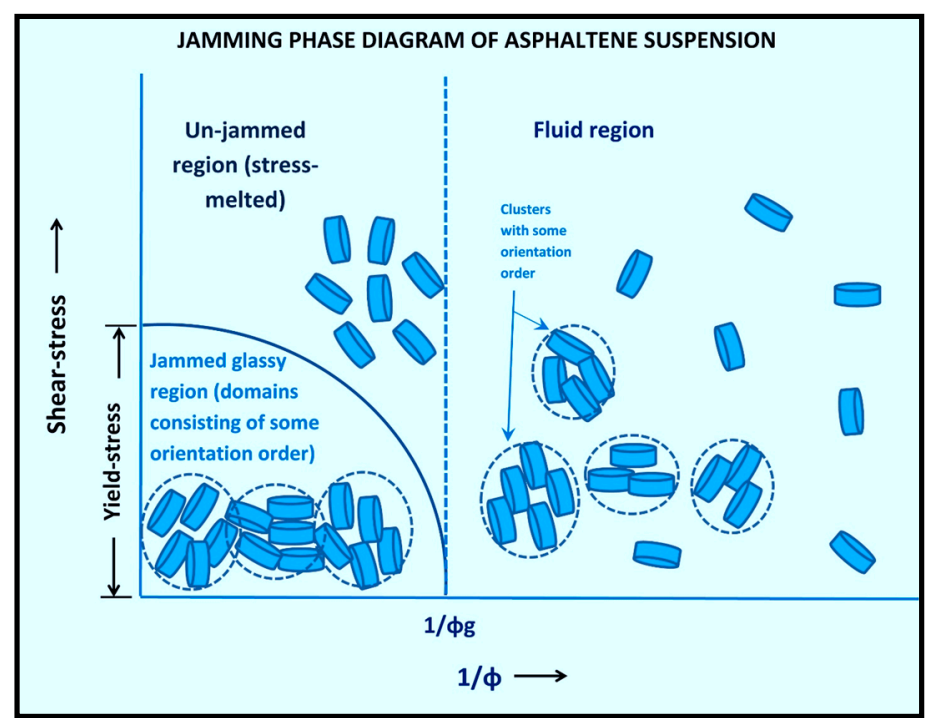

Figure 6. Non-equilibrium jamming phase diagram of suspension.

\section{Modelling of the Viscosity of Asphaltene Suspensions}

\subsection{Virial Expansion of the Relative Viscosity of Suspension}

The virial expansion of the relative viscosity of suspension $\left(\eta_{r}\right)$, defined as the ratio of suspension viscosity $(\eta)$ to continuous-phase viscosity $\left(\eta_{C}\right)$, is as follows:

$$
\eta_{r}=1+B \phi+C \phi^{2}+D \phi^{3}+\ldots \ldots
$$

where $B, C, D$ are virial coefficients. For infinitely dilute suspensions, only the following truncated form of the virial expansion is sufficient:

$$
\eta_{r}=1+B \phi
$$

For very dilute suspensions, this equation can be rearranged as: 


$$
1-\left(1 / \eta_{r}\right)=B \phi
$$

Thus, the virial coefficient $B$ of a suspension can be determined experimentally from the slope of the plot of $1-\left(1 / \eta_{r}\right)$ versus $\phi$ data at low values of $\phi$.

\subsection{Determination of Intrinsic Viscosity and Solvation of Particles from Virial Coefficient}

For infinitely dilute suspensions, the relative viscosity of a suspension is expressed in terms of the Einstein equation as:

$$
\eta_{r}=1+[\eta] \phi
$$

where $[\eta]$ is the intrinsic viscosity of the suspension. When the suspension particles are solvated, Equation (4) needs to be modified as:

$$
\eta_{r}=1+[\eta]_{S} \phi_{S}
$$

where $[\eta]_{S}$ is the intrinsic viscosity of solvated particles and $\phi_{S}$ is the effective volume fraction of the solvated particles. Upon comparison of Equation (5) with Equation (2), it follows that:

$$
B=[\eta]_{S}\left(\phi_{S} / \phi\right)=[\eta]_{S} k_{S}
$$

where $k_{S}$ is the solvation coefficient defined as the ratio of the effective (solvated) volume fraction to actual (un-solvated) volume fraction of particles. When the particles are solid and spherical, then the intrinsic viscosity is 2.5 for solvated or un-solvated particles. Therefore, experimental virial coefficient determined from the slope, as discussed in the preceding section, can be used to determine the solvation coefficient of particles as follows:

$$
k_{S}=B / 2.5
$$

The solvation coefficient $k_{S}$ for solid spherical particles can further be related to the thickness of the solvation layer $(\delta)$ as:

$$
k_{S}=\phi_{S} / \phi=\left(1+\frac{\delta}{R_{o}}\right)^{3}
$$

where $R_{o}$ is the radius of the unsolvated particle.

For disk-shaped particles, the intrinsic viscosity varies with the aspect ratio $(r)$ (defined as the ratio of disk thickness to disk diameter). The intrinsic viscosity $[\eta]$ of randomly-oriented disk-shaped particles is given as [14]:

$$
[\eta]=\frac{5}{2}+\frac{32}{15 \pi}\left(\frac{1}{r}-1\right)-0.628\left[\frac{1-r}{1-(0.075 r)}\right]
$$

For solvated disk-shaped particles, the intrinsic viscosity can be expressed as:

$$
[\eta]_{S}=\frac{5}{2}+\frac{32}{15 \pi}\left(\frac{1}{r_{S}}-1\right)-0.628\left[\frac{1-r_{S}}{1-\left(0.075 r_{S}\right)}\right]
$$

where $r_{S}$ is the aspect ratio of solvated disk-shaped particles. From Equation (6), it follows that:

$$
B=[\eta]_{S} k_{S}=k_{S}\left\{\frac{5}{2}+\frac{32}{15 \pi}\left(\frac{1}{r_{S}}-1\right)-0.628\left(\frac{1-r_{S}}{1-\left(0.075 r_{S}\right)}\right)\right\}
$$

In what follows, a simple model is developed to relate the solvation coefficient $k_{S}$ to the aspect ratio $r_{S}$ of solvated asphaltene nanoaggregates. As noted in Figure 3, the asphaltene nanoaggregates consist of a dense aromatic core of diameter $3.64 \mathrm{~nm}$ and a highly aliphatic mobile shell of thickness $1.44 \mathrm{~nm}$. The thickness of the disk is about $0.67 \mathrm{~nm}$. We assume that the solvation of these disk 
"core-shell" type particles occurs in the aliphatic shell portion of the composite particle. The solvated portion of the shell is expected to be immobile whereas the un-solvated portion of the shell is assumed to be "free-draining" meaning that the continuous-phase fluid can flow freely in the un-solvated portion of the shell. Figure 7 shows a schematic representation of a solvated disk-type particle. Let $n$ be the number density of particles, that is, the number of particles per unit volume of the suspension. The dry and solvated volumes of a single particle are:

$$
\begin{gathered}
V_{d r y}=\pi R_{o}^{2} t \\
V_{\text {solvated }}=\pi R^{2} t
\end{gathered}
$$

The solvation coefficient $k_{S}$ can be expressed as:

$$
k_{S}=\phi_{S} / \phi=n V_{\text {solvated }} / n V_{d r y}=\left(\frac{R}{R_{o}}\right)^{2}
$$

The aspect ratios of the particle based on the core and solvated diameters are:

$$
\begin{aligned}
& r_{o}=t / 2 R_{o} \\
& r_{S}=t / 2 R
\end{aligned}
$$

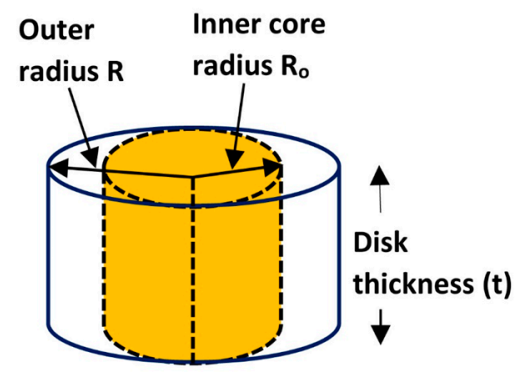

Figure 7. Schematic representation of a solvated asphaltene nanoaggregate.

Combining Equations (14)-(16) gives the following relation for the solvation coefficient:

$$
k_{S}=\left(\frac{r_{o}}{r_{S}}\right)^{2}
$$

From Equations (11) and (17), it follows that:

$$
B=\left\{\frac{5}{2}+\frac{32}{15 \pi}\left(\frac{1}{r_{S}}-1\right)-0.628\left(\frac{1-r_{S}}{1-\left(0.075 r_{S}\right)}\right)\right\}\left(\frac{r_{o}}{r_{S}}\right)^{2}
$$

The only unknown in this equation is $r_{S}$, the aspect ratio of the solvated asphaltene nanoaggregate. The virial coefficient $B$ is determined from the experimental viscosity data for dilute suspensions. The aspect ratio $r_{o}$ based on the core diameter is 0.184 (ratio of $0.67 \mathrm{~nm}$ to $3.64 \mathrm{~nm}$ ). Thus, Equation (18) can be used to determine the aspect ratio $r_{S}$ of solvated asphaltene nanoaggregates.

It is important to note that this approach for the estimation of aspect ratio $r_{S}$ of solvated asphaltene nanoaggregates using Equation (18), where the dependence of both intrinsic viscosity and solvation coefficient on $r_{S}$ is taken into consideration, is novel and has not been reported elsewhere. Once the aspect ratio $r_{S}$ is known, the intrinsic viscosity of solvated nanoaggregates can be determined from Equation (10) and the solvation coefficient can be determined from Equation (17). 
Based on the dimensions of the asphaltene nanoaggregate, the following ranges for $r_{S}, k_{S}$, and $[\eta]_{S}$ are expected: $0.103 \leq r_{S} \leq 0.184,1.0 \leq k_{S} \leq 3.21$, and $4.99 \leq[\eta]_{S} \leq 7.86$. The solvation layer thickness is assumed to vary from $0 \mathrm{~nm}$ to $1.44 \mathrm{~nm}$ corresponding to the aliphatic shell present on the periphery of a dense aromatic core.

\subsection{Viscosity Model}

Consider a suspension of solvated particles. The relative viscosity of such a suspension in the limit of dilute range is given by the well-known Einstein equation (Equation (5)). The intrinsic viscosity of solvated particles is dependent on three factors: shape of the solvated particle, aspect ratio of the solvated particle, and rotary Peclet number defined as:

$$
P e=\eta_{C} R^{3} \dot{\gamma} / k_{B} T
$$

where $\dot{\gamma}$ is the shear rate, $R$ is the radius of the solvated particle, $k_{B}$ is the Boltzmann constant, and $T$ is the absolute temperature of the suspension. For spherical particles, solvated or un-solvated, the intrinsic viscosity has a constant value of 2.5 independent of the Peclet number. For non-spherical disk-shaped asphaltene nanoaggregates, the intrinsic viscosity in the limit of low Peclet numbers (small shear rate and small disk radius) is dependent only on the aspect ratio and is given by Equation (10).

The limitation of the Einstein equation, Equation (5), is that it is valid only in the limit $\phi_{S} \rightarrow 0$. Equation (5) could be rewritten as:

$$
\eta_{r}=1+[\eta]_{S}\left(\frac{\text { Volume of solvated particles }}{\text { Total volume of suspension }}\right) \phi_{S}
$$

Pal [15] has argued that the Einstein equation could be extended to non-dilute suspensions provided that the "free volume" available to particles is used instead of the "total volume of suspension" in Equation (20). It is the free volume available to the particles that is more relevant in determining the viscosity of non-dilute suspensions. Thus:

$$
\eta_{r}=1+[\eta]_{S}\left(\frac{\text { Volume of solvated particles }}{\text { Free volume available to suspension }}\right) \phi_{S}
$$

From Equation (21), it follows that:

$$
\eta_{r}=1+[\eta]_{S}\left(\frac{\phi_{S}}{1-\phi_{S}}\right)
$$

This equation is expected to be applicable at low to moderate concentration of particles. At high concentrations, clustering of particles dominates. When clusters are formed, they trap a significant amount of continuous-phase fluid leading to an increase in the effective dispersed phase concentration. At glass transition concentration $\phi_{g}$, the entire suspension becomes jammed as a single large cluster. In the jammed or arrested state of the suspension, the relative viscosity of the suspension diverges.

Thus, we propose the following model to describe the viscosity data of suspensions of asphaltene nanoaggregates:

$$
\eta_{r}=1+[\eta]_{S}\left(\frac{\phi_{e f f}}{1-\phi_{e f f}}\right)
$$

where $\phi_{e f f}$ is the effective volume fraction of the solvated particles. Pal $[15,16]$ recently proposed and tested a number of empirical expressions for the effective volume fraction of particles to account for the 
clustering and jamming of particles in nanosuspensions of spherical particles. The following empirical expression for the effective volume fraction of particles was found to be quite successful:

$$
\phi_{e f f} / \phi_{S}=\left[1+\left(\frac{1-\phi_{g}}{\phi_{g}}\right)\left(\sqrt{1-\left(\frac{\phi_{g}-\phi_{S}}{\phi_{g}}\right)^{2}}\right)\right]
$$

This expression for $\phi_{e f f}$ meets the following necessary conditions:

(a) When $\phi_{S} \rightarrow 0, \phi_{e f f}=\phi_{S}$, that is, no clustering of particles occurs.

(b) When $\phi_{S} \rightarrow \phi_{g}, \phi_{e f f}=1$, that is, the suspension is jammed as a single large cluster.

(c) The slope of ratio $\phi_{e f f} / \phi_{S}$ with respect to $\phi_{S}$ is always positive, that is, $\partial\left(\phi_{e f f} / \phi_{S}\right) / \partial \phi_{S} \geq 0$. The ratio $\phi_{e f f} / \phi_{S}$ can only increase with the increase in $\phi_{S}$ due to an increase in the size of the clusters.

(d) At high values of $\phi_{S}, \phi_{e f f} / \phi_{S}$ becomes constant and in the limit $\phi_{S} \rightarrow \phi_{g}, \partial\left(\phi_{e f f} / \phi_{S}\right) / \partial \phi_{S}=0$.

Figure 8 shows the influence of clustering of solvated particles on the effective volume fraction of the dispersed-phase $\phi_{e f f}$. The plot is generated from Equation (24) assuming that the solvated particles are spherical in shape and the glass transition volume fraction of solvated particles is 0.58 . As reflected in the figure, the difference in the effective volume fraction and actual volume fraction of solvated particles, that is, $\phi_{e f f}-\phi_{S}$, increases with the increase in $\phi_{S}$. At the glass transition concentration where the suspension is jammed as a single large cluster, the effective volume fraction of the dispersed-phase becomes unity $\left(\phi_{e f f}=1.0\right)$.

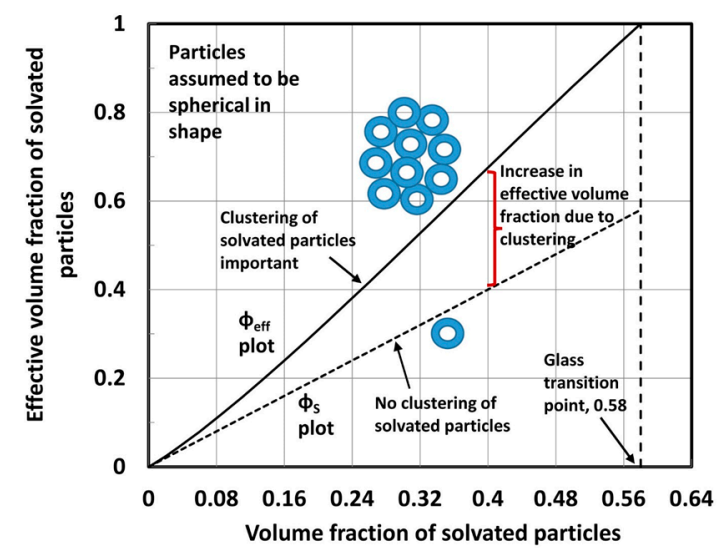

Figure 8. Influence of clustering of solvated particles on the effective volume fraction of dispersed-phase.

\section{Analysis and Scaling of Experimental Viscosity Data for Asphaltene Suspensions}

\subsection{Experimental Viscosity Data for Asphaltene Suspensions}

A large pool of experimental viscosity data (twenty sets) on asphaltene suspensions available in the literature are considered. The details of the data are summarized in Table 1 . The data pool covers broad ranges of temperature and volume fraction of asphaltenes. Asphaltenes from different sources are used to prepare the suspensions. The types of solvents used in the preparation of suspensions are also varied. Figure 9 shows the plots of relative viscosity versus volume fraction of asphaltenes for the twenty sets of data described in Table 1. A large variation in the relative viscosity is observed for a given asphaltene volume fraction due to variations in the type of asphaltenes, source of asphaltenes, type of solvent, and temperature. 


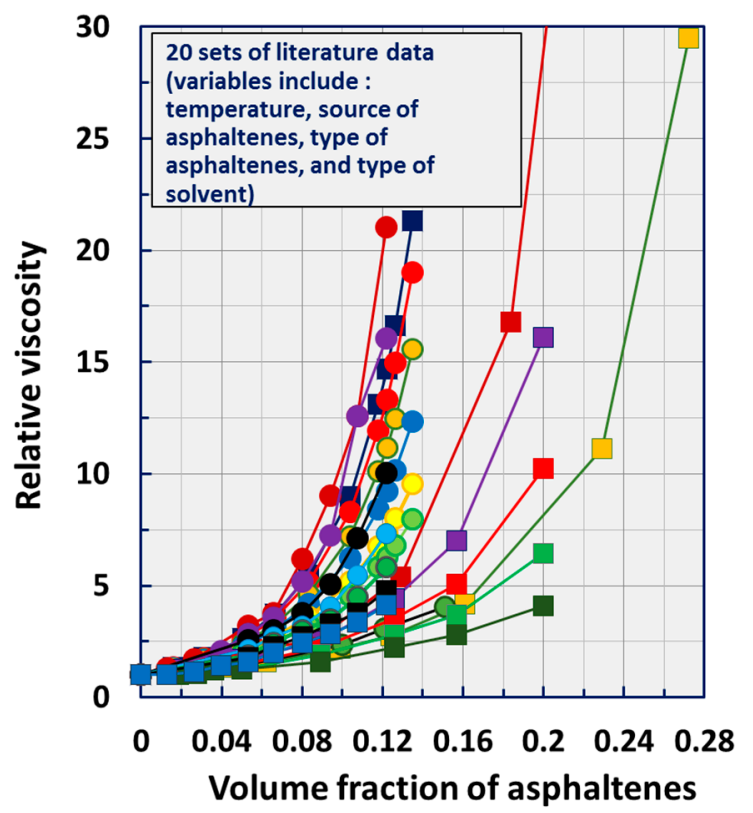

Figure 9. Twenty sets of experimental relative viscosity data gathered from different sources in the literature.

Table 1. Twenty sets of viscosity data on asphaltene suspensions taken from different sources.

\begin{tabular}{|c|c|c|c|c|c|c|}
\hline $\begin{array}{l}\text { Set } \\
\text { No. }\end{array}$ & Reference & $\begin{array}{c}\text { Source of } \\
\text { Asphaltenes }\end{array}$ & $\begin{array}{c}\text { Type of } \\
\text { Asphaltenes }\end{array}$ & Solvent & $\begin{array}{c}\text { Temperature } \\
\left({ }^{\circ} \mathrm{C}\right)\end{array}$ & $\begin{array}{c}\text { Range of } \\
\text { Asphaltene } \\
\text { Volume } \\
\text { Fraction }(\phi)\end{array}$ \\
\hline 1 & Sheu et al. [17] & $\begin{array}{l}\text { Ratawi } \\
\text { crude }\end{array}$ & $\begin{array}{c}\text { IC7 } \\
\text { (C7 insoluble) }\end{array}$ & toluene & 25 & $0-0.28$ \\
\hline 2 & Barre et al. [18] & $\begin{array}{c}\text { Saudi } \\
\text { Arabian } \\
\text { crude oil }\end{array}$ & $\begin{array}{c}\text { IC7 } \\
\text { (C7 insoluble) }\end{array}$ & toluene & 25 & $0-0.22$ \\
\hline $3-8$ & Luo and Gu [19] & $\begin{array}{c}\text { Lloydminster } \\
\text { Canadian } \\
\text { heavy oil }\end{array}$ & $\begin{array}{c}\text { IC5 } \\
\text { (C5 insoluble) }\end{array}$ & maltenes & $20-60$ & $0-0.135$ \\
\hline 9 & Bouhadda et al. [20] & $\begin{array}{l}\text { Algerian } \\
\text { crude }\end{array}$ & $\begin{array}{c}\text { IC7 } \\
\text { (C7 insoluble) }\end{array}$ & toluene & 25 & $0-0.15$ \\
\hline $10-13$ & Eyssautier et al. [4] & $\begin{array}{l}\text { Safaniya } \\
\text { vacuum } \\
\text { residue }\end{array}$ & $\begin{array}{c}\text { IC5 } \\
\text { (C5 insoluble) }\end{array}$ & maltenes & 80-200 & $0-0.20$ \\
\hline $14-20$ & Ghanavati et al. [21] & $\begin{array}{c}\text { Iranian } \\
\text { heavy oil }\end{array}$ & $\begin{array}{c}\text { IC6 } \\
\text { (C6 insoluble) }\end{array}$ & maltenes & $25-85$ & $0-0.122$ \\
\hline
\end{tabular}

\subsection{Estimation of Intrinsic Viscosity and Solvation Coefficient}

The intrinsic viscosity and solvation coefficient of different asphaltene suspensions are obtained from the experimental values of the virial coefficient $B$. The virial coefficient is obtained from the slope of the plot of $1-\left(1 / \eta_{r}\right)$ versus $\phi$ data at low values of $\phi$. Figure 10 shows the typical plots of $1-\left(1 / \eta_{r}\right)$ versus $\phi$ data at low values of $\phi$. As expected, the plots of $1-\left(1 / \eta_{r}\right)$ versus $\phi$ data are linear for low values of $\phi$. The data are generally linear for $\phi<0.05$. It is possible that some clustering of asphaltene nanoaggregates takes when $\phi<0.05$. However, the number density of clusters is expected to be negligible as compared with the number density of individual nanoaggregates at these low concentrations. The virial coefficients obtained for different sets of data are summarized in Table 2. The aspect ratio $\left(r_{S}\right)$ of solvated asphaltene nanoaggregates are calculated from Equation (18). Once the 
aspect ratio is known, the intrinsic viscosity and solvation coefficient are determined from Equations (10) and (17), respectively. The values of $[\eta]_{S}$ and $k_{S}$ for different sets of asphaltene suspensions are given in Table 2. The values of $r_{S}, k_{S}$, and $[\eta]_{S}$ are within the ranges specified in Section 3.2.

Figure 11 shows the variations of $[\eta]_{S} /[\eta]_{C O R E}$ and $k_{S}$ with the aspect ratio $r_{S}$ where $[\eta]_{C O R E}=$ 4.99 corresponding to the core (un-solvated asphaltene nanoaggregate) aspect ratio of 0.184 . The solvation coefficient $k_{S}$ as well as $[\eta]_{S} /[\eta]_{\text {CORE }}$ decrease with the increase in the aspect ratio $r_{S}$. This is to be expected as an increase in $r_{S}$ corresponds to a decrease in the solvation of asphaltene nanoaggregate and a decrease in the diameter of the nanoaggregate. Interestingly, the decrease in the solvation coefficient is much larger than the decrease in $[\eta]_{S} /[\eta]_{\text {CORE }}$ with the increase in $r_{S}$.

Table 2. Summary of virial coefficients, aspect ratios, intrinsic viscosities, $[\eta]_{S} /[\eta]_{C O R E}$ and solvation coefficients for twenty sets of asphaltene suspensions.

\begin{tabular}{cccccc}
\hline Set No. & Virial Coefficient & Aspect Ratio $\left(r_{S}\right)$ & Intrinsic Viscosity & {$[\eta]_{S} /[\eta]_{\text {CORE }}$} & Solvation Coefficient $\left(k_{S}\right)$ \\
\hline 1 & 6.5 & 0.167 & 5.35 & 1.072 & 1.215 \\
2 & 9.0 & 0.148 & 5.85 & 1.172 & 1.538 \\
3 & 15 & 0.124 & 6.76 & 1.355 & 2.219 \\
4 & 14.5 & 0.125 & 6.69 & 1.341 & 2.167 \\
5 & 13.5 & 0.128 & 6.56 & 1.287 & 1.058 \\
6 & 12.5 & 0.132 & 6.42 & 1.242 & 1.774 \\
7 & 11.0 & 0.138 & 6.20 & 1.214 & 1.683 \\
8 & 10.2 & 0.142 & 6.06 & 1.05 & 1.145 \\
9 & 6.0 & 0.172 & 5.24 & 1.144 & 1.436 \\
10 & 8.2 & 0.154 & 5.71 & 1.062 & 1.179 \\
11 & 6.25 & 0.17 & 5.30 & 1.026 & 1.074 \\
12 & 5.5 & 0.178 & 5.12 & 1.417 & 1.00 \\
13 & 4.99 & 0.184 & 4.99 & 1.381 & 2.475 \\
14 & 17.5 & 0.117 & 7.07 & 1.303 & 2.322 \\
15 & 16.0 & 0.121 & 6.89 & 1.239 & 2.000 \\
16 & 13.0 & 0.13 & 6.50 & 1.204 & 1.780 \\
17 & 11.0 & 0.138 & 6.18 & 1.174 & 1.664 \\
18 & 10.0 & 0.143 & 6.01 & 1.114 & 1.536 \\
19 & 9.0 & 0.149 & 5.86 & 5.56 & 1.349 \\
20 & 7.5 & 0.159 & & & \\
\hline
\end{tabular}
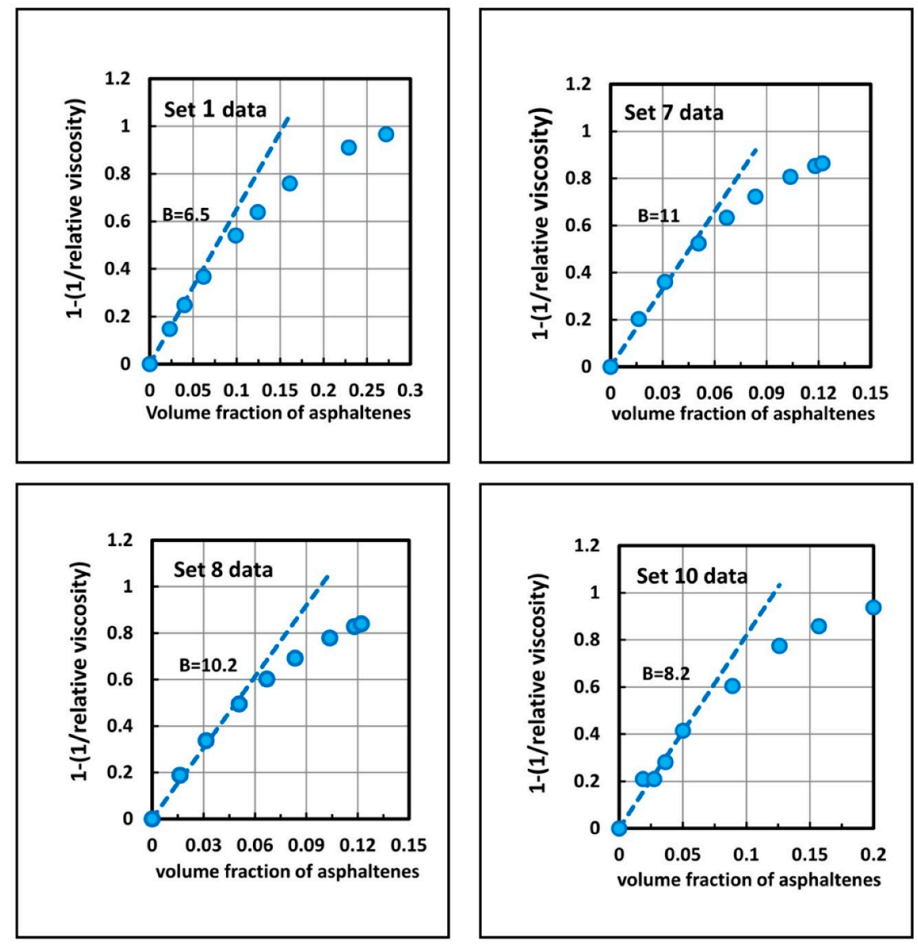

Figure 10. Estimation of virial coefficient $B$ of asphaltene suspensions. 


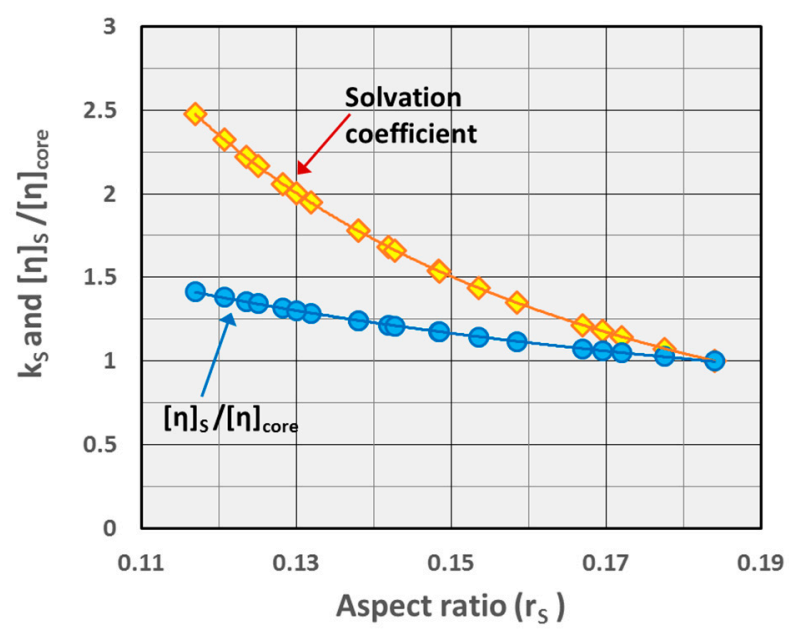

Figure 11. Variations of $k_{S}$ and $[\eta]_{S} /[\eta]_{C O R E}$ with the aspect ratio $r_{S}$.

\subsection{Scaling of Relative Viscosity of Asphaltene Suspensions}

According to the viscosity model discussed in Section 3.3, the relative viscosity of suspension of solvated asphaltene nanoaggregates can be expressed as:

$$
\eta_{r}=1+[\eta]_{S} f\left(\phi_{S}\right)
$$

where $f\left(\phi_{S}\right)$ is a function of $\phi_{S}$ (volume fraction of solvated nanoaggregates). Provided the intrinsic viscosity $[\eta]_{S}$ is constant from one suspension system to another, the relative viscosity of different asphaltene suspensions should scale on a $\phi_{S}$ basis. As observed in Figure 11, $[\eta]_{S}$ variation from one suspension system to another is not large. Therefore, an attempt is made to scale the relative viscosity data of asphaltene suspensions with respect to $\phi_{S}$.

Figure 12 shows the scaling of relative viscosity of asphaltene suspensions with respect to $\phi_{S}$. Despite some variation of $[\eta]_{S}$ from one suspension system to another, the scaling is remarkably successful. The wide variation in the relative viscosities observed in Figure 9 disappears and the data of different asphaltene suspensions collapse on to a single curve.

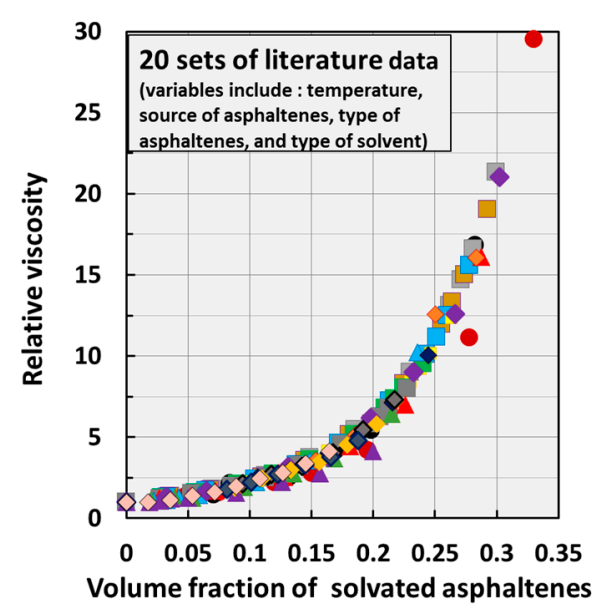

Figure 12. Scaling of relative viscosity of 20 different sets of asphaltene suspensions with respect to $\phi_{S}$, volume fraction of solvated asphaltene nanoaggregates. 


\subsection{Comparison of Model Prediction with Experimental Data}

The viscosity model proposed in Section 3.3 (Equation (23) in conjunction with Equation (24)) could be rearranged as:

$$
\left(\eta_{r}-1\right) /[\eta]_{S}=f\left(\phi_{S}\right)=\left(\frac{\phi_{e f f}}{1-\phi_{e f f}}\right)
$$

According to this equation, the exact scaling law is $\left(\eta_{r}-1\right) /[\eta]_{S} \alpha \phi_{S}$. This way the variation of $[\eta]_{S}$ from one suspension system to another is taken care of. Figure 13 shows the viscosity data of 20 sets of asphaltene suspensions plotted as $\left(\eta_{r}-1\right) /[\eta]_{S}$ versus $\phi_{S}$. The plot confirms the scaling law $\left(\eta_{r}-1\right) /[\eta]_{S} \propto \phi_{S}$. All the data collapses on to a single curve. Furthermore, the data can be described remarkably well by the viscosity model described by Equation (23) in conjunction with Equation (24) using the glass transition volume fraction $\phi_{g}$ of 0.395 . The viscosity data tends to diverge at $\phi_{g}$ of 0.395 . This can be seen more clearly in Figure 14 where the data are plotted on a semi-log scale. Interestingly, according to Wensink and Lekkerkerker [9], the columnar crystalline order becomes stable in suspensions of platelets beyond a $\phi$ value of approximately 0.40 . However, it should be emphasized that more experimental viscosity data are needed at high concentrations of asphaltenes in order to confirm this value of glass transition.

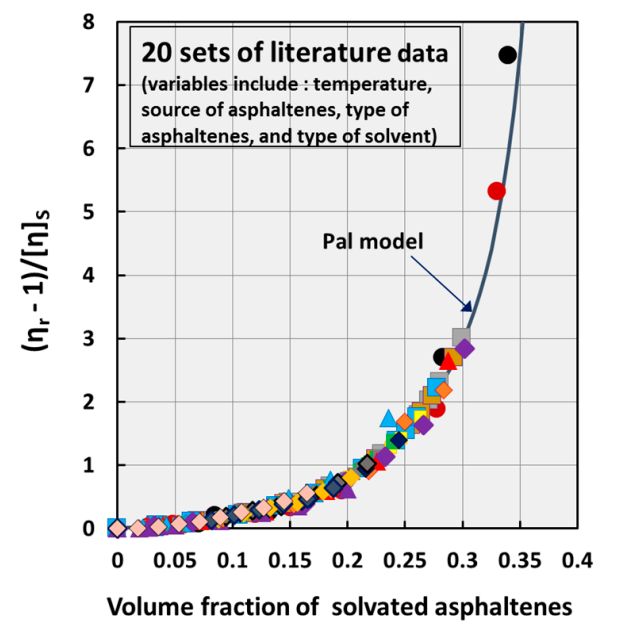

Figure 13. Twenty viscosity datasets of asphaltene suspensions plotted as $\left(\eta_{r}-1\right) /[\eta]_{S}$ versus $\phi_{S}$.

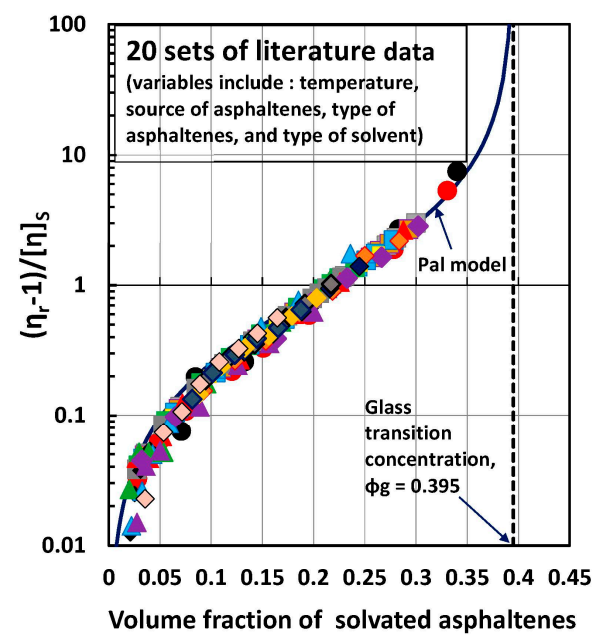

Figure 14. Twenty viscosity datasets of asphaltene suspensions plotted as $\left(\eta_{r}-1\right) /[\eta]_{S}$ versus $\phi_{S}$ on a semi-log scale. 


\section{Conclusions}

Most recent SANS, SAXS, and solid-state ${ }^{1} \mathrm{H}$ NMR studies of asphaltenes available in the literature indicate that asphaltene nanoaggregates are disk-shaped "core-shell" particles consisting of a dense aromatic core and a mobile aliphatic shell. In this work, the viscosity of suspensions of asphaltene nanoaggregates are modelled based on this structure of nanoaggregates. The solvation and clustering of nanoaggregates, and the jamming of the suspension at glass transition volume fraction of asphaltene nanoaggregates are all considered in the scaling analysis and modeling of the suspension viscosity. New expressions are developed to estimate the aspect ratio and solvation coefficient of nanoaggregates from the experimental values of the virial coefficient of viscosity expansion of suspension. The predicted values of aspect ratio $\left(r_{S}\right)$, solvation coefficient $\left(k_{S}\right)$, and intrinsic viscosity $\left([\eta]_{S}\right)$ from the model fall in the following ranges: $0.103 \leq r_{S} \leq 0.184,1.0 \leq k_{S} \leq 3.21$, and $4.99 \leq[\eta]_{S} \leq 7.86$. Twenty sets of experimental viscosity data on asphaltene suspensions gathered from different sources are analyzed. The variation in the relative viscosity of asphaltene suspensions from one system to another is due to variation in the degree of solvation of asphaltene nanoaggregates. The experimental values of $r_{S}, k_{S}$, and $[\eta]_{S}$ fall in the following ranges: $0.117 \leq r_{S} \leq 0.184$, $1.0 \leq k_{S} \leq 2.475$, and $4.99 \leq[\eta]_{S} \leq 7.07$; these ranges are consistent with the model predictions. A new scaling law is discovered in this work for the viscosity of asphaltene suspensions. The scaling law and the proposed model describe the experimental viscosity data of a variety of different asphaltene suspensions remarkably well. The jamming of the asphaltene suspension is predicted to occur at a volume fraction of solvated asphaltene nanoaggregates of approximately 0.4 . The viscosity of new asphaltene systems can be predicted from the proposed model over a broad range of asphaltene concentrations provided that the intrinsic viscosity of the suspension is known. The intrinsic viscosity of asphaltene suspensions can be estimated from the experimental viscosity data for dilute suspensions.

Acknowledgments: Financial support from NSERC is appreciated.

Conflicts of Interest: The author declares no conflict of interest.

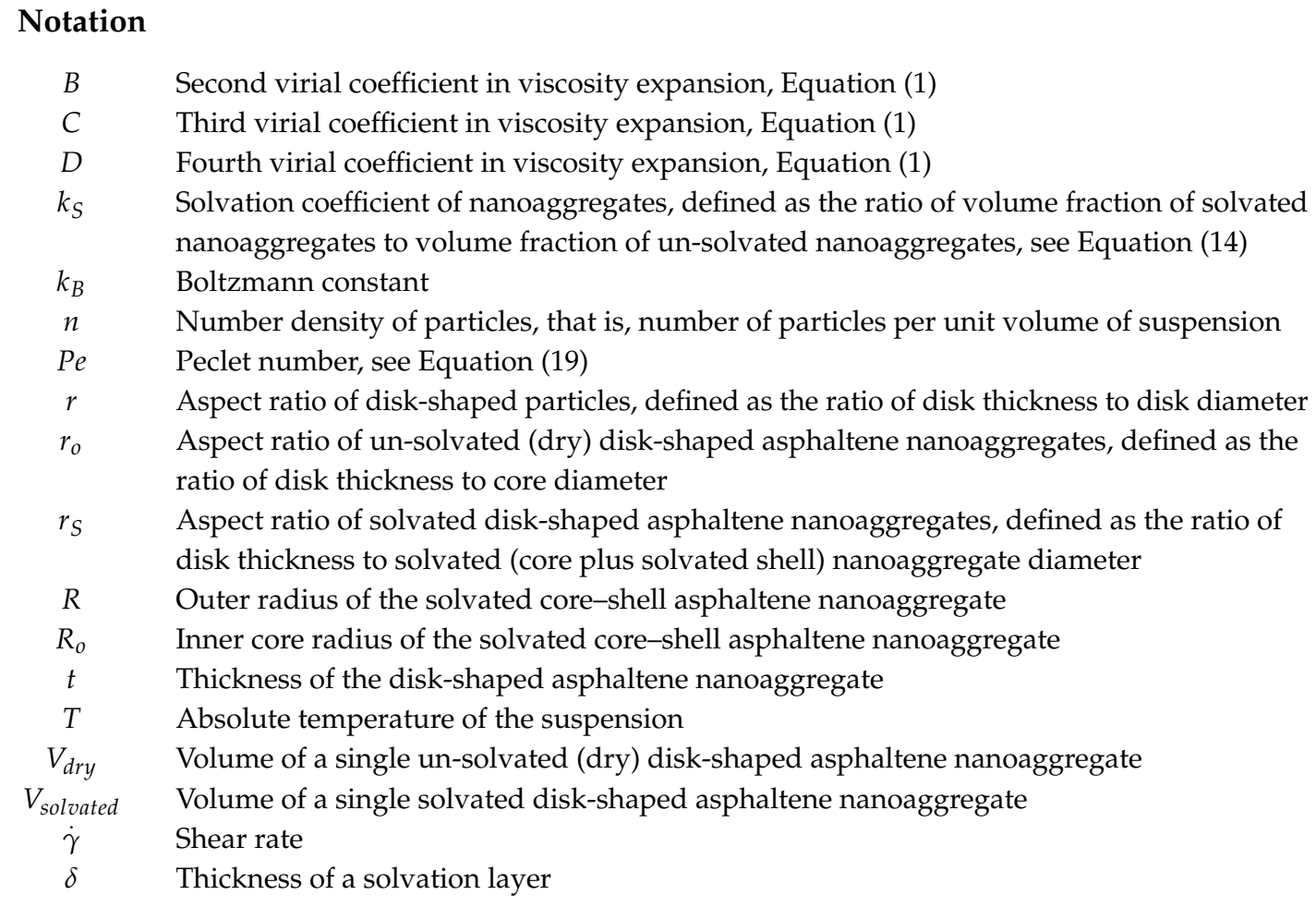




$\begin{array}{cl}\eta & \text { Viscosity of suspension } \\ \eta_{C} & \text { Continuous-phase viscosity } \\ \eta_{r} & \text { Relative viscosity of suspension } \\ {[\eta]} & \text { Intrinsic viscosity of suspension } \\ {[\eta]_{C O R E}} & \text { Intrinsic viscosity of suspension of un-solvated asphaltene nanoaggregates } \\ {[\eta]_{S}} & \text { Intrinsic viscosity of suspension of solvated asphaltene nanoaggregates } \\ \phi & \text { Volume fraction of dispersed-phase (particles) } \\ \phi_{C} & \text { Volume fraction of particles where the entire suspension becomes ordered columnar } \\ & \text { crystalline (see Figure 5) } \\ \phi_{e f f} & \text { Effective volume fraction of solvated nanoaggregates, given by Equation (24). The effective } \\ & \text { volume fraction takes into account both solvation of individual nanoaggregates and } \\ & \text { continuous-phase immobilization due to clustering of solvated nanoaggregates } \\ \phi_{g} & \text { The glass transition concentration of suspension of solvated disk-shaped asphaltene } \\ & \text { nanoaggregates } \\ \phi_{I N} & \text { Volume fraction of disk-shaped particles where transition occurs from isotropic liquid } \\ & \text { region to nematic region (see Figure 5) } \\ \phi_{N C} & \text { Volume fraction of disk-shaped particles where transition occurs from nematic region to } \\ & \text { nematic + crystal coexistence region (see Figure 5) } \\ \phi_{S} & \text { Volume fraction of solvated disk-shaped nanoaggregates without taking into consideration } \\ & \text { any clustering of nanoaggregates }\end{array}$

\section{References}

1. Majumdar, R.D.; Montina, T.; Mullins, O.C.; Gerken, M.; Hazendonk, P. Insights into asphaltene aggregate structure using ultrafast MAS solid-state ${ }^{1} \mathrm{H}$ NMR spectroscopy. Fuel 2017, 193, 359-368. [CrossRef]

2. Mullins, O.C.; Sabbah, H.; Eyssautier, J.; Pomerantz, A.E.; Barré, L.; Andrews, A.B.; Ruiz-Morales, Y.; Mostowfi, F.; McFarlane, R.; Goual, L.; et al. Advances in asphaltene science and the Yen-Mullins model. Energy Fuels 2012, 26, 3986-4003. [CrossRef]

3. Eyssautier, J.; Levitz, P.; Espinat, D.; Jestin, J.; Gummel, J.; Grillo, I.; Barrie, L. Insight into asphaltene nanoaggregate structure inferred by small angle neutron and X-ray scattering. J. Phys. Chem. B 2011, 115, 6827-6837. [CrossRef] [PubMed]

4. Eyssautier, J.; Henaut, I.; Levitz, P.; Espinat, D.; Barre, L. Organization of asphaltenes in a vacuum residue: A small-angle X-ray scattering (SAXS) - Viscosity approach at high temperatures. Energy Fuels 2012, 26, 2696-2704. [CrossRef]

5. Eyssautier, J.; Frot, D.; Barre, L. Structure and dynamic properties of colloidal asphaltene aggregates. Langmuir 2012, 28, 11997-12004. [CrossRef] [PubMed]

6. Goual, L. Petroleum asphaltenes. In Crude Oil Emulsions-Composition Stability and Characterization; El-Sayed, M., Raouf, A., Eds.; In Tech: Rijeka, Croatia, 2012; pp. 27-42.

7. Pal, R.; Vargas, F. On the interpretation of viscosity data of suspensions of asphaltene nano-aggregates. Can. J. Chem. Eng. 2014, 92, 573-577. [CrossRef]

8. Pal, R. A new model for the viscosity of asphaltene solutions. Can. J. Chem. Eng. 2015, 93, 747-755. [CrossRef]

9. Wensink, H.H.; Lekkerkerker, H.N.W. Phase diagram of hard colloidal platelets: A theoretical account. Mol. Phys. 2009, 107, 2111-2118. [CrossRef]

10. Frenkel, D.; Mulder, B.M.; McTague, J.P. Phase diagram of a system of hard ellipsoids. Phys. Rev. Lett. 1984, 52, 287-290. [CrossRef]

11. De Michele, C.; Schilling, R.; Sciortino, F. Dynamics of uniaxial hard ellipsoids. Phys. Rev. Lett. 2007, 98, 265702-1-265702-4. [CrossRef] [PubMed]

12. Ginzburg, V.V.; Balazs, A.C. Calculating phase diagrams of polymer-platelet mixtures using density functional theory: Implications for polymer/clay composites. Macromolecules 1999, 32, 5681-5688. [CrossRef]

13. Van der Kooij, F.M.; Lekkerkerker, H.N.W. Formation of nematic liquid crystals in suspensions of hard colloidal platelets. J. Phys. Chem. B 1998, 102, 7829-7832. [CrossRef]

14. Van der Kooij, F.M.; Boek, E.S.; Philipse, A.P. Rheology of dilute suspensions of hard platelike colloids. J. Colloid Interface Sci. 2001, 235, 344-349. [CrossRef] [PubMed] 
15. Pal, R. Viscosity-concentration relationships for nanodispersions based on glass transition point. Can. J. Chem. Eng. 2017, in press. [CrossRef]

16. Pal, R. Modeling the viscosity of concentrated nanoemulsions and nanosuspensions. Fluids 2016, 1, 11. [CrossRef]

17. Sheu, E.Y.; Storm, D.A.; De Tar, M.M. Asphaltenes in polar solvents. J. Non-Cryst. Solids 1991, 131-133, 341-347. [CrossRef]

18. Barre, L.; Simon, S.; Palermo, T. Solution properties of asphaltenes. Langmuir 2008, 24, 3709-3717. [CrossRef] [PubMed]

19. Luo, P.; Gu, Y. Effects of asphaltene content on the heavy oil viscosity at different temperatures. Fuel 2007, 86, 1069-1078. [CrossRef]

20. Bouhadda, Y.; Bendedouch, D.; Sheu, E.; Krallafa, A. Some preliminary results on a physico-chemical characterization of a Hassi Messaoud petroleum asphaltene. Energy Fuels 2000, 14, 845-853. [CrossRef]

21. Ghanavati, M.; Shojaei, M.J.; Ramazani, A.S.A. Effects of asphaltene content and temperature on viscosity of Iranian heavy crude oil: Experimental and modeling study. Energy Fuels 2013, 27, 7217-7232. [CrossRef]

(C) 2017 by the author. Licensee MDPI, Basel, Switzerland. This article is an open access article distributed under the terms and conditions of the Creative Commons Attribution (CC BY) license (http://creativecommons.org/licenses/by/4.0/). 